\title{
Compliance with clinical practice guidelines for breast cancer treatment: a population-based study of quality-of-care indicators in Italy
}

Carlotta Sacerdote ${ }^{1,6^{*}}$, Rita Bordon², Sabina Pitarella ${ }^{2}$, Maria Piera Mano ${ }^{2}$, Ileana Baldi ${ }^{1}$, Denise Casella ${ }^{2}$, Daniela Di Cuonzo ${ }^{1}$, Alfonso Frigerio ${ }^{3}$, Luisella Milanesio ${ }^{3}$, Franco Merletti ${ }^{1}$, Eva Pagano ${ }^{1}$, Fulvio Ricceri ${ }^{4}$, Stefano Rosso ${ }^{2}$, Nereo Segnan², Mariano Tomatis ${ }^{2}$, Giovannino Ciccone ${ }^{1}$, Paolo Vineis ${ }^{4,5}$ and Antonio Ponti ${ }^{2}$

\begin{abstract}
Background: It has been documented that variations exist in breast cancer treatment despite wide dissemination of clinical practice guidelines. The aim of this population-based study was to evaluate the impact of regional guidelines (Piedmont guidelines, PGL) for breast cancer diagnosis and treatment on quality-of-care indicators in the Northwestern Italian region of Piedmont.

Methods: We included two samples of women aged 50-69 years with incident breast cancer treated in Piedmont before and after the introduction of PGL: 600 in 2002 (pre-PGL) and 621 in 2004 (post-PGL). Patients were randomly selected among all incident breast cancer cases identified through the hospital discharge records database. We extracted clinical data on breast cancer cases from medical charts and ascertained vital status through linkage with town offices. We assessed compliance with 14 quality-of-care indicators from PGL recommendations, before and after their introduction in clinical practice.

Results: Among patients with invasive lesions, $77.1 \%(N=368)$ and $77.5 \%(N=383)$ in the pre-PGL and post-PGL groups, respectively, received breast conservative surgery (BCS) as a first-line treatment. Following BCS, $87.7 \%$ received radiotherapy in 2002, compared to $87.9 \%$ in 2004. Of all patients at medium-to-high risk of distant metastasis, 65.5\% ( $N=268)$ and 63.6\% ( $N=252)$ received chemotherapy in 2002 and in 2004, respectively. Among the 117 patients with invasive lesions and negative estrogen receptor status in 2002, hormonal therapy was prescribed in 23 of them (19.6\%). The incorrect prescription of hormonal therapy decreased to $10.8 \%(N=10)$ among the 92 estrogen receptor-negative patients in $2004(p<0.01)$.

Compliance with PGL recommendations was already high in the pre-PGL group, although some quality-of-care indicators did not reach the standard. In the pre/post analysis, 8 out of 14 quality-of-care indicators showed an improvement from 2002 to 2004, but only 4 out of 14 reached statistical significance. We did not find any change in the risk of mortality in the post-PGL versus the pre-PGL group (adjusted hazard ratio 0.94, 95\% Cl 0.56-1.56).
\end{abstract}

Conclusions: These results highlight the need to continue to improve breast cancer care and to measure adherence to PGL.

Keywords: Breast cancer, Guideline adherence, Population-based, Evidence-based medicine, Quality of care

\footnotetext{
* Correspondence: carlotta.sacerdote@cpo.it

${ }^{1}$ Cancer Epidemiology Unit, San Giovanni Battista Hospital, CPO Piemonte

and University of Turin, Turin, Italy

${ }^{6}$ Cancer Epidemiology Unit, San Giovanni Battista Hospital, Via Santena 7,

10129, Torino, Italy

Full list of author information is available at the end of the article
} 


\section{Background}

Evidence-based guidelines serve as a tool to ensure that patients receive treatment based on the best available evidence. In 1995, Sainsbury et al. postulated that the improvement of clinical practice in breast cancer treatment could increase 5-year survival by up to 10\% [1].

In the Piedmont Region (Northwestern Italy, population 4.25 million), clinical practice guidelines (PGL) for the treatment of breast cancer were first released in July 2002 and disseminated to all relevant clinicians and other stakeholders [2,3] Furthermore, as from 1996, the Piedmont Region has been covered by a breast screening program for all resident women aged 50-69 years.

It has been well documented that there is considerable variation in breast cancer treatment despite wide dissemination of clinical practice guidelines [4]. Variations are usually related not only to patient characteristics, such as age and educational level, but also to geographic area of residence and hospital and physician characteristics [5].

Although several studies have examined the different surgical and medical breast cancer treatments employed following the publication of clinical practice guidelines, to our knowledge few reports have included a comparison with clinical practice prior to guideline publication, and even fewer have examined the impact of guidelines in clinical practice at a population level [6,7].

To evaluate the real impact of PGL on breast cancer treatment in Piedmont, we collected data from the medical charts of women with breast cancer aged 50-69 years to assess compliance with 14 quality-of-care indicators, based on PGL recommendations, before and after the introduction of PGL in clinical practice. Furthermore, we explored the use of post-surgical medical treatment, including radiotherapy, chemotherapy and hormonal therapy in the two periods, according to FIGO stage, lymph node involvement and hormone receptor status. Finally, we compared the survival rates of patients treated before and after the introduction of PGL.

\section{Methods}

\section{Population and data sources}

The Piedmont hospital discharge records (HDR) database was used to identify female patients with incident breast cancer, aged 50-69 years, residing in Piedmont and surgically treated in 2002 and 2004 at regional hospitals. In 2002 we identified 1,764 female patients, of whom 866 underwent surgical treatment in the first 6 months of 2002. Among these 866 patients, 600 were randomly selected for this study (pre-PGL group). In 2004, 1,777 female patients were identified, of whom 905 underwent surgical treatment in the first 6 months of 2004. Among those 905 patients, 621 were randomly selected for this study (post-PGL group) (Figure 1). The start of the post-PGL period was 1.5 years after the introduction of PGL, as at this time they were likely to have been implemented in Piedmont hospitals.

We did not include prevalent breast cancer cases, i.e., women who had been previously hospitalized for breast cancer and were recorded in the Piedmont Cancer Registry and/or were in the HDR database between 1998 and 2002.

For the purposes of this study, breast cancer was defined by the following International Classification of Diseases $9^{\text {th }}$ Revision - Clinical Modification (ICD9-CM) codes: 174, carcinoma of the breast; 233.0, in situ carcinoma of the breast; 238.3, neoplasm of uncertain behavior of the breast; or 239.3, neoplasm of unspecified nature of the breast, in any position of the HDR database.

Fifty-eight patients from the randomly-selected prePGL group and 71 patients from the randomly-selected post-PGL group were not eligible for inclusion as they had benign lesions, prevalent lesions, recurrences, other cancers, no surgical treatment, or unavailable clinical records. After exclusion, 542 patients were left in the pre-PGL group and 550 in the post PGL-group, and were included in the following analyses (Figure 1).

In situ carcinomas of the breast were over-sampled in both groups. To do this, the same inclusion criteria were applied, but were not restricted to the first 6 months of 2002 and 2004. Instead, all women with in situ carcinoma (ICD9-CM code 233.0) who were surgically treated at any time in 2002 and 2004 were included. The total number of oversampled patients with in situ lesions was 121 in 2002 and 108 in 2004. This over-sampling was only used in the analyses concerning the indicators for patients with carcinoma in situ (CIS) in order to increase the power of the study.

The accuracy of the method adopted to identify incident breast cancer cases was validated using data from the Piedmont Cancer Registry, which covers about 20\% of the regional population [8], as a gold standard. The sensitivity of the algorithm was $76.7 \%$ for breast cancer and the positive predictive value was $92.6 \%$.

Follow-up of patients from both groups was performed through linkage to different databases using two hierarchical linkage keys. The HDR database was used to identify all subsequent relevant hospitalizations (2002-2005) for other surgical treatments (on the same, or other lesions), medical complications and chemotherapy sessions. Radiotherapy was assessed through linkage with the radiotherapy outpatient record database (which also includes extraregional radiotherapy records), and hormonal therapy was assessed though linkage to the pharmaceutical prescription record database (which includes all drug prescriptions reimbursed to patients by the public health system).

All clinical records of any surgical or radiotherapy hospitalization identified for patients in the HDR 


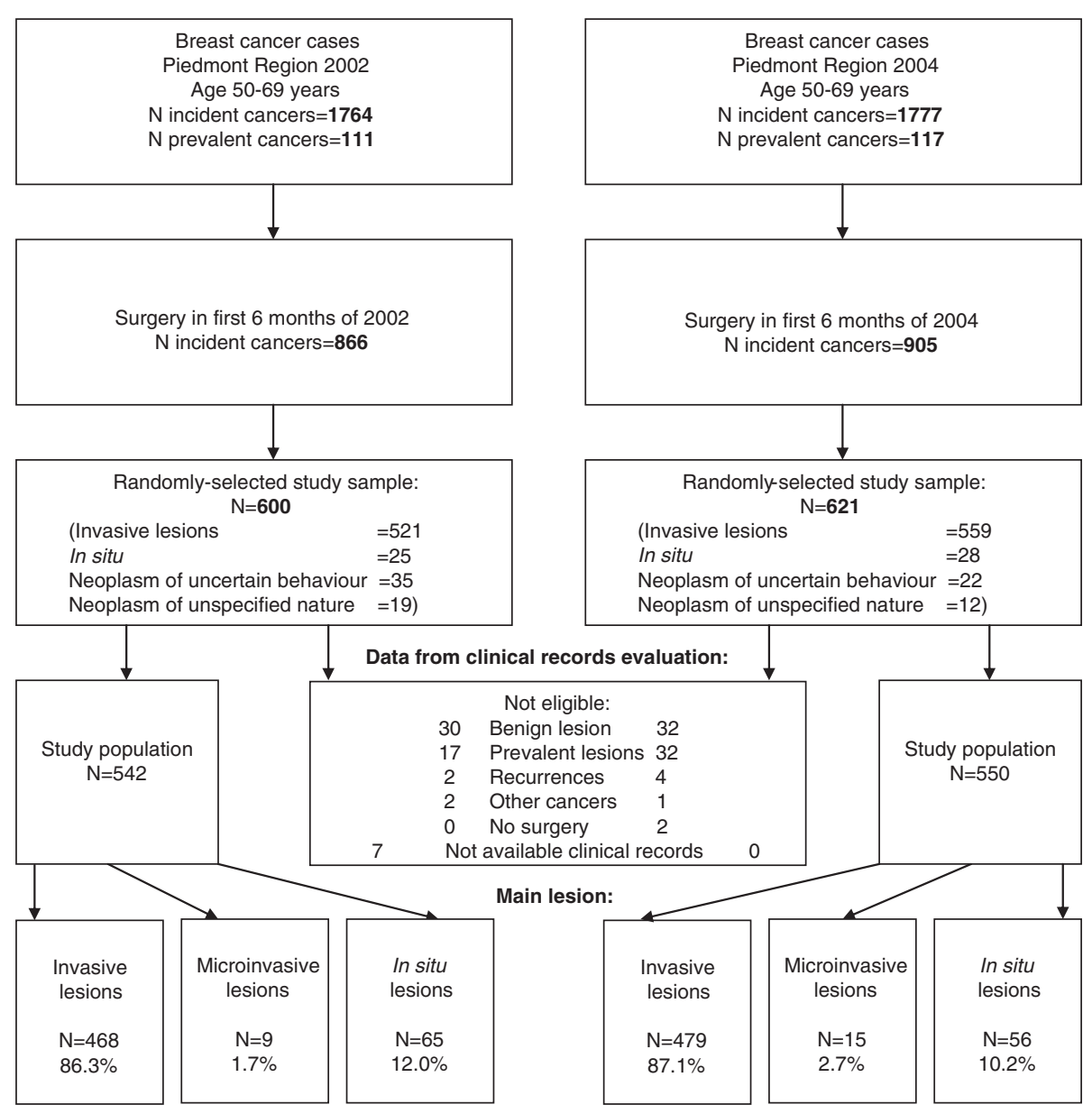

Figure 1 Study population of women with incident breast cancer who underwent surgery in 2002 and 2004.

database were retrieved. The clinical data were extracted from medical charts by two breast cancer screening technicians supervised by a gynecologist and an epidemiologist, working independently of the practitioners caring for patients in different hospitals. It was impossible to extract data blinded to the year of treatment since the data were obtained directly from the patients' records. To control the extracted data, a random $10 \%$ sample was rechecked by the supervisors, blinded to the previous decisions. The collected data were entered into a database previously used for clinical audit (the Audit System on Quality of Breast Cancer Treatment), developed by a multidisciplinary team from the European Breast Cancer Network [9].

The ascertainment of vital status was carried out through linkage with town offices, identifying the date of death and allowing for the retrieval of the death certificate to identify the specific causes of death (2002-2010). All procedures concerning death certificates, data collection and coding were applied uniformly to both groups.

\section{Main outcome measures}

Fourteen quality-of-care indicators were chosen to evaluate the impact of PGL (Table 1). We explored the use of the sentinel lymph node (SLN) technique by surgical unit annual case load, to measure the introduction of this procedure. Finally, we investigated post-surgical medical treatment, including chemotherapy among patients with invasive lesions at low/medium to high risk of distant metastasis (according to the Goldhirsch scheme) [10], and hormonal therapy prescribed according to estrogen receptor status.

\section{Statistical analyses}

The differences in distribution between post-PGL and pre-PGL breast cancer cases according to patient, tumor and surgical unit characteristics and in the rates of patients receiving post-surgical medical treatment were assessed by two-way Chi square Test or Fisher Exact Test.

The 14 quality-of-care indicators (Table 1) were analyzed as dichotomous variables by multivariable logistic regression models, using the period (post-PGL versus pre-PGL) as the main effect and controlling for 
Table 1 Piedmont Region clinical practice guidelines quality-of-care indicators

\begin{tabular}{lcc}
\hline $\begin{array}{l}\text { Level of evidence (AHRQ } \\
\text { grade of underlying }\end{array}$ & Quality-of-care indicators & Function \\
recommendation) & & Quality-of- \\
care & standards
\end{tabular}

$\%$ of patients with invasive lesions treated with axillary clearance or SLN technique

$\%$ of patients treated with axillary clearance with $>9$ lymph nodes

$\%$ with NO dissection among CIS patients

\% correct SLN identification

$\%$ histopathological grading available

\% hormonal receptor availability

$\%$ immediate reconstruction after mastectomy with BCS
$\%$ radiotherapy in patients treated

AHRQ grade of underlying recommendation (reference http://archive.ahrq.gov/clinic/epcarch.htm), BCS: breast conservative surgery, pT1: small primary tumor, SLN: sentinel lymph node, CIS: carcinoma in situ.
Calculates the proportion of patients diagnosed with invasive lesions of a pathological size of $\leq 20 \mathrm{~mm}$ (pT1, microinvasive included), not clinically multicentric or multifocal, who were treated with BCS.

Calculates the proportion of BCS (last BCS if more than one) for invasive or in situ lesions which ensured clear margins (distance $>1 \mathrm{~mm}$ from the lesion), out of the total number of BCS performed.

Calculates the proportion of patients whose first surgical treatment was not followed by further local operations that were required due to incomplete excision (excluding failed biopsies), out of the total number of patients who were surgically treated for localized invasive or in situ lesions with a positive or suspicious cytological or histological pre-operative diagnosis.

Calculates the proportion of patients surgically treated for invasive lesions (excluding microinvasive lesions) of a maximum pathological size $\leq 10 \mathrm{~mm}$ for which there was no frozen section, out of the total number of patients with the same diagnosis.

Calculates the proportion of patients with invasive lesions who were treated with axillary dissection or SLN technique, out of the total number of patients with invasive lesions.

Calculates the proportion of patients with invasive lesions who were treated with axillary clearance (level I-III), excluding sampling, and from whom at least 10 lymph nodes were excised, out of the total number of patients with invasive lesions who were treated with axillary clearance.

Calculates the proportion of patients diagnosed with CIS or not otherwise specified in situ lesions (microinvasive cancer excluded) on whom no axillary dissection was performed (not even level I) out of the total number of patients with this diagnosis who were surgically treated.

Calculates the\% of SLN identified out of the total of identified SLN in patients with invasive lesions, who were treated with SLN technique.

Calculates the proportion of patients with invasive lesions (excluding microinvasive cancer) who were surgically treated and for whom measuring was provided, out of the total number of patients with invasive lesions who underwent surgical treatment

Calculates the proportion of patients with invasive lesions (excluding microinvasive cancer) who were surgically treated and for whom measuring was provided, out of the total number of patients with invasive lesions who underwent surgical treatment.

Calculates the proportion of patients with invasive or in situ tesions who had mastectomies and immediate reconstruction, out of the total number of patients treated with mastectomy.

Calculates the proportion of patients who were treated with BCS E invasive or in situ lesions, and for whom radiotherapy followed, out of the total number of patients with the same diagnosis who were treated with BCS.

Calculates the proportion of patients with invasive lesions and positive estrogen receptors who received hormonal therapy. 
Table 2 Distribution of breast cancer cases according to various patient, tumor and surgical unit characteristics, 2002 and 2004, Piedmont Region, Italy

\begin{tabular}{|c|c|c|c|}
\hline & \multicolumn{2}{|c|}{$\mathrm{N}$ and $\%$ of patients } & \multirow{2}{*}{$\begin{array}{l}\text { Effect of year } \\
\text { ( } p \text { value) }\end{array}$} \\
\hline & 2002 & 2004 & \\
\hline Sample size (N) & $542(49.6 \%)$ & $550(50.4 \%)$ & \\
\hline \multicolumn{4}{|l|}{ Age (years) } \\
\hline $50-54$ & $134(24.7 \%)$ & $124(22.5 \%)$ & \multirow[t]{5}{*}{0.35} \\
\hline $55-59$ & $111(20.5 \%)$ & $122(22.2 \%)$ & \\
\hline $60-64$ & $166(30.6 \%)$ & $151(27.5 \%)$ & \\
\hline $65-69$ & $131(24.2 \%)$ & $153(27.8 \%)$ & \\
\hline Missing & 0 & 0 & \\
\hline \multicolumn{4}{|l|}{ Educational level } \\
\hline Bachelor & 69 (12.7\%) & 99 (18.0\%) & \multirow[t]{5}{*}{0.11} \\
\hline Secondary & $151(27.9 \%)$ & $146(26.5 \%)$ & \\
\hline Professional & $222(40.9 \%)$ & 197 (35.8\%) & \\
\hline Intermediate and primary & $85(15.7 \%)$ & $88(16.0 \%)$ & \\
\hline None and unknown & $15(2.8 \%)$ & $20(3.6 \%)$ & \\
\hline \multicolumn{4}{|l|}{ Pathological T stage } \\
\hline In Situ & $62(11.4 \%)$ & $55(10.0 \%)$ & \multirow[t]{15}{*}{0.54} \\
\hline $1 \mathrm{mic}$ & $10(1.8 \%)$ & $15(2.7 \%)$ & \\
\hline 1 & $4(0.7 \%)$ & $5(0.9 \%)$ & \\
\hline $1 a$ & 17 (3.2\%) & $25(4.5 \%)$ & \\
\hline $1 b$ & $75(13.8 \%)$ & $85(15.5 \%)$ & \\
\hline $1 c$ & $186(34.3 \%)$ & $189(34.4 \%)$ & \\
\hline 2 & $147(27.2 \%)$ & $136(24.7 \%)$ & \\
\hline 3 & $5(0.9 \%)$ & $10(1.8 \%)$ & \\
\hline 4 & $5(0.9 \%)$ & $5(0.9 \%)$ & \\
\hline $4 a$ & $3(0.6 \%)$ & $0(0 \%)$ & \\
\hline $4 b$ & $18(3.3 \%)$ & $15(2.7 \%)$ & \\
\hline $4 c$ & $1(0.2 \%)$ & $0(0 \%)$ & \\
\hline $4 d$ & $2(0.4 \%)$ & $2(0.4 \%)$ & \\
\hline$x$ & $4(0.7 \%)$ & $2(0.4 \%)$ & \\
\hline Missing & $3(0.6 \%)$ & $6(1.1 \%)$ & \\
\hline \multicolumn{4}{|l|}{ Pathological N stage } \\
\hline 0 & $205(43.3 \%)$ & $158(31.3 \%)$ & \multirow[t]{13}{*}{$<0.01$} \\
\hline Sentinel lymph node & $80(16.9 \%)$ & 167 (33.1\%) & \\
\hline 1 & $21(4.4 \%)$ & 37 (7.3\%) & \\
\hline $1 a$ & $20(4.2 \%)$ & 72 (14.3\%) & \\
\hline $1 b$ & $7(1.5 \%)$ & $3(0.6 \%)$ & \\
\hline 1b1 & $40(8.4 \%)$ & $2(0.4 \%)$ & \\
\hline $1 \mathrm{~b} 2$ & $7(1.5 \%)$ & $3(0.6 \%)$ & \\
\hline $1 b 3$ & $56(11.8 \%)$ & $3(0.6 \%)$ & \\
\hline $1 \mathrm{~b} 4$ & $11(2.3 \%)$ & $3(0.6 \%)$ & \\
\hline 2 & 15 (3.2\%) & $25(5.0 \%)$ & \\
\hline 3 & 0 & $23(4.6 \%)$ & \\
\hline$x$ & $9(1.9 \%)$ & $6(1.2 \%)$ & \\
\hline Missing & $2(0.4 \%)$ & $2(0.4 \%)$ & \\
\hline
\end{tabular}


Table 2 Distribution of breast cancer cases according to various patient, tumor and surgical unit characteristics, 2002 and 2004, Piedmont Region, Italy (Continued)

\begin{tabular}{|c|c|c|c|}
\hline \multicolumn{4}{|l|}{ Pathological TNM stage } \\
\hline In situ & $65(12.0 \%)$ & $56(10.2 \%)$ & 0.04 \\
\hline । & $189(34.8 \%)$ & $233(42.3 \%)$ & \\
\hline$\| \mathrm{A}$ & $127(23.4 \%)$ & $112(20.4 \%)$ & \\
\hline$\| B$ & $75(13.8 \%)$ & $53(9.6 \%)$ & \\
\hline III or more & $47(8.8 \%)$ & $65(11.9 \%)$ & \\
\hline Missing & $39(7.2 \%)$ & $31(5.6 \%)$ & \\
\hline \multicolumn{4}{|l|}{ Grading (invasive only) } \\
\hline Low & $58(12.0 \%)$ & $82(16.5 \%)$ & 0.09 \\
\hline Intermediate & $69(14.4 \%)$ & $86(17.4 \%)$ & \\
\hline High & $103(21.5 \%)$ & $89(18.4 \%)$ & \\
\hline Not performed and missing & 250 & 238 & \\
\hline \multicolumn{4}{|c|}{ Disease detected through regional screening program } \\
\hline Yes & $208(38.4)$ & $236(42.9 \%)$ & 0.001 \\
\hline No, symptomatic & 209 (38.6\%) & $171(31.9 \%)$ & \\
\hline No, asymptomatic & $110(20.3 \%)$ & $112(20.4 \%)$ & \\
\hline Missing & $15(2.8 \%)$ & $31(5.6 \%)$ & \\
\hline \multicolumn{4}{|l|}{ Surgical unit annual case load } \\
\hline$<50$ & $124(22.9 \%)$ & $126(22.9 \%)$ & 0.001 \\
\hline 50-149 & 249 (45.9\%) & $250(45.5 \%)$ & \\
\hline$\geq 150$ & $155(28.6 \%)$ & $147(26.8 \%)$ & \\
\hline Missing & 14 (2.6\%) & 27 (4.8\%) & \\
\hline
\end{tabular}

confounders (age, educational level, clinical stage, screening provenience and surgical unit annual case load). The results are presented as frequencies, adjusted odds ratios $\left(\mathrm{OR}_{\mathrm{adj}}\right)$ and $95 \%$ confidence intervals $(95 \% \mathrm{CI})$ estimated from logistic models to measure the probability of achieving standards in 2004 compared to 2002. We performed Cox proportional hazards regression analyses to study survival. Proportional hazard assumptions were tested with the Grambsch and Therneau test before analysis. Statistical analyses were performed using SAS 8.2 and STATA v10.

\section{Results}

\section{Patient characteristics}

Among the women included in the analyses, the distribution by type of lesion in the pre-PGL group was: $88.0 \%$ invasive or microinvasive ( $\mathrm{N}=477$ ) (ICD9-CM code 174), and $12.0 \%$ in situ $(\mathrm{N}=65)$ (ICD9-CM code 233.0); in the post-PGL group it was $89.8 \%$ invasive or microinvasive $(\mathrm{N}=494)$, and $10.2 \%$ in situ $(\mathrm{N}=56)$ (non-statistically significant difference) (Table 1$)$.

Table 2 shows the distribution of breast cancer cases by patient, tumor and care provider characteristics for the prePGL and post-PGL groups. Both groups had similar age and educational level distribution. In contrast, there was a statistically significant difference in the distribution of clinical stages between 2002 and 2004. The proportion of cases diagnosed through the regional screening program increased from $38.4 \%(\mathrm{~N}=208)$ in 2002 to $42.9 \%(\mathrm{~N}=236)$ in 2004 ( $\mathrm{p}=0.001)$. Of all patients diagnosed with CIS, the percent diagnosed by the regional screening program increased from $45.9 \%(\mathrm{~N}=28)$ in 2002 , to $63.6 \%(\mathrm{~N}=35)$ in 2004 (data not shown). The annual case load of surgical units was stable over the two periods (Table 2).

\section{Surgical and medical treatment}

In the pre-PGL group $77.1 \%(\mathrm{~N}=368)$ of patients with invasive lesions received BCS as a first-line treatment, and for $62.7 \%(\mathrm{~N}=299)$ of patients in the pre-PGL group, BCS was a definitive surgical treatment. In the post-PGL group the numbers were $77.5 \%(\mathrm{~N}=383)$ and $66.6 \%(\mathrm{~N}=329)$, respectively (Figure 2).

As for post-surgical medical treatment, following BCS, $87.7 \%(\mathrm{~N}=341)$ of the pre-PGL group and $87.9 \%$ $(\mathrm{N}=362)$ of the post-PGL group received radiotherapy alone, or in combination with chemotherapy (Figure 3). Post-surgical treatment with adjuvant chemotherapy was received by $50.9 \%(\mathrm{~N}=276)$ of the pre-PGL group (about 


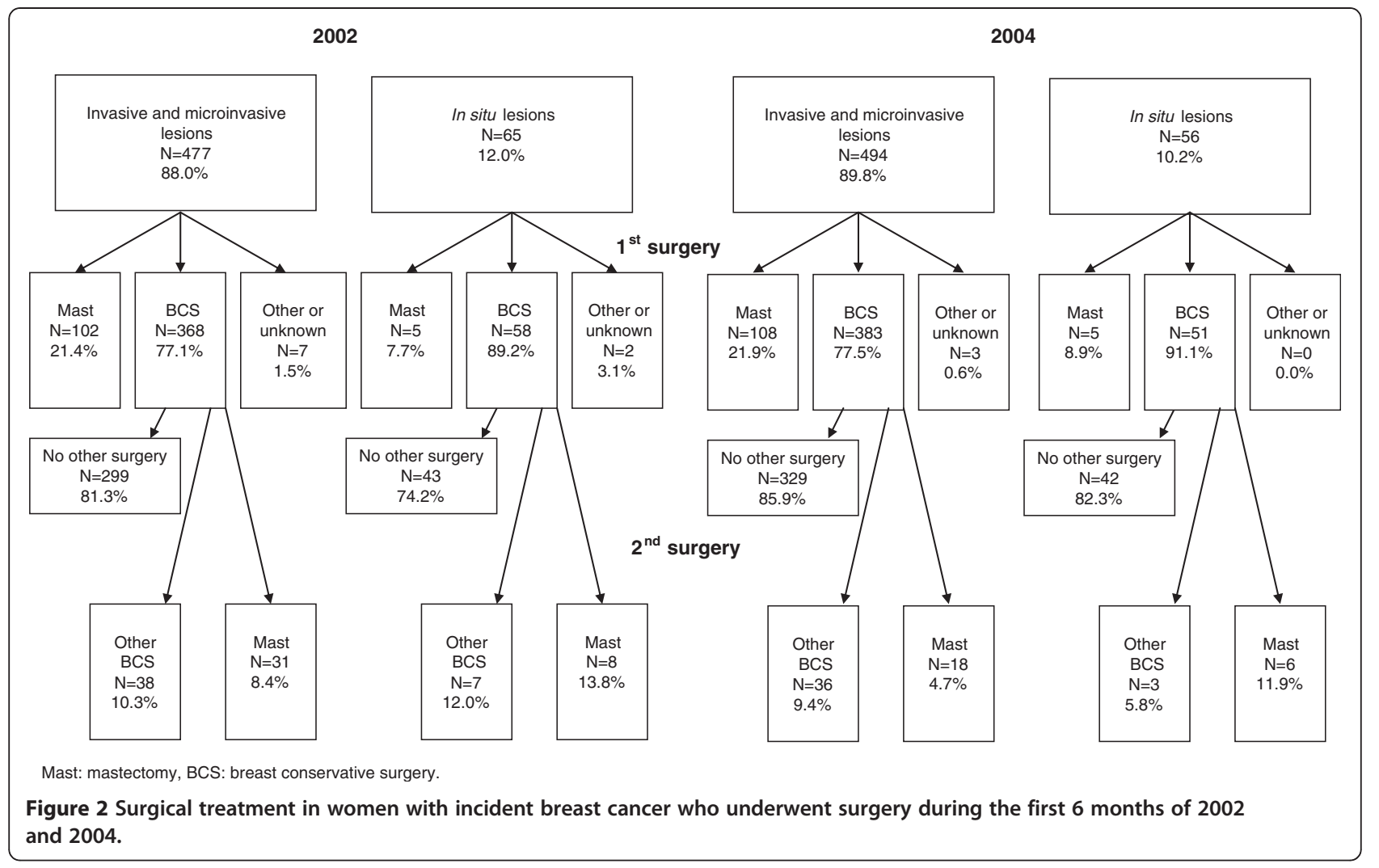

$46 \%$ following BCS and 67\% following mastectomy) and by $46.2 \%(\mathrm{~N}=254)$ of the post-PGL group (about 39\% following BCS and 69\% following mastectomy) (data not shown).

Post-surgical medical treatment of invasive lesions is shown in Tables 3 and 4 . Of all patients with invasive lesions at medium-to-high risk of distant metastasis, $64.6 \%$ received chemotherapy $(\mathrm{N}=520): 65.5 \%$ in the prePGL group and $63.6 \%$ in the post-PGL group. Among 113 women with invasive lesions at low risk of distant metastasis $14.3 \%$ and $1.4 \%$ received chemotherapy in 2002 and 2004, respectively (Table 3).

Hormonal therapy was prescribed to 684 out of 901 (75.9\%) patients with invasive lesions. Of women who were prescribed hormonal therapy, 4.8\% $(\mathrm{N}=33)$ had negative estrogen receptor status. The incorrect prescription of hormonal therapy in patients with negative receptor status decreased from 19.6\% $(\mathrm{N}=23)$ in 2002 to $10.8 \%(\mathrm{~N}=10)$ in $2004(\mathrm{p}<0.01)$ (Table 4$)$.

\section{Compliance with PGL recommendations}

Univariate analysis showed that compliance with PGL recommendations was already high in the pre-PGL group. Indeed, five of nine quality-of-care indicators did not achieve the proposed standard (\% of malignant lesions with cytological or histological pre-operative diagnosis, \% frozen section in lesions $\leq 10 \mathrm{~mm}, \%$ of patients treated with axillary clearance with $>9$ lymph nodes, \% with no dissection among CIS patients, \% radiotherapy in patients treated with BCS).

In the pre/post analysis, studying the probability of achieving standards in 2004 compared to 2002, although eight of the 14 examined quality-of-care indicators changed in the expected direction, only four indicators improved substantially from 2002 to 2004: percent of malignant lesions with cytological or histological diagnosis before surgery $\left(\mathrm{OR}_{\mathrm{adj}} 0.64,95 \% \mathrm{CI}\right.$ 0.49-0.85), percent of BCS in pT1 lesions and percent of BCS performed with free margins $\left(\mathrm{OR}_{\mathrm{adj}}\right.$ 0.41, 95\% CI $0.22-0.75$ and $\mathrm{OR}_{\mathrm{adj}} 0.65,95 \% \mathrm{CI}$ 0.41-1.01 respectively) and percentage of frozen section in lesions $\leq 10 \mathrm{~mm}\left(\mathrm{OR}_{\mathrm{adj}} 0.32,95 \% \mathrm{CI} 0.16-0.65\right)$. The other indicators were stable in the two periods (Table 5).

The number and percent of women treated with SLN technique by surgical unit annual case load is shown in Table 6. The PGL recommendations said that the SLN technique should be performed, as an alternative to axillary dissection, only by surgical units with a high annual case load. The percent of patients who were treated with SLN technique by a surgical unit with an annual case load of $<50$ (based on the total number of women who underwent surgery in low-case load units) was $19.1 \%$ in $2002(\mathrm{~N}=25)$ and $42.3 \%$ in $2004(\mathrm{~N}=55)$. There was a big increase from 2002 to 2004 in the use of this technique across all strata of annual surgical case load, but we observed the biggest increase in the lowest category 


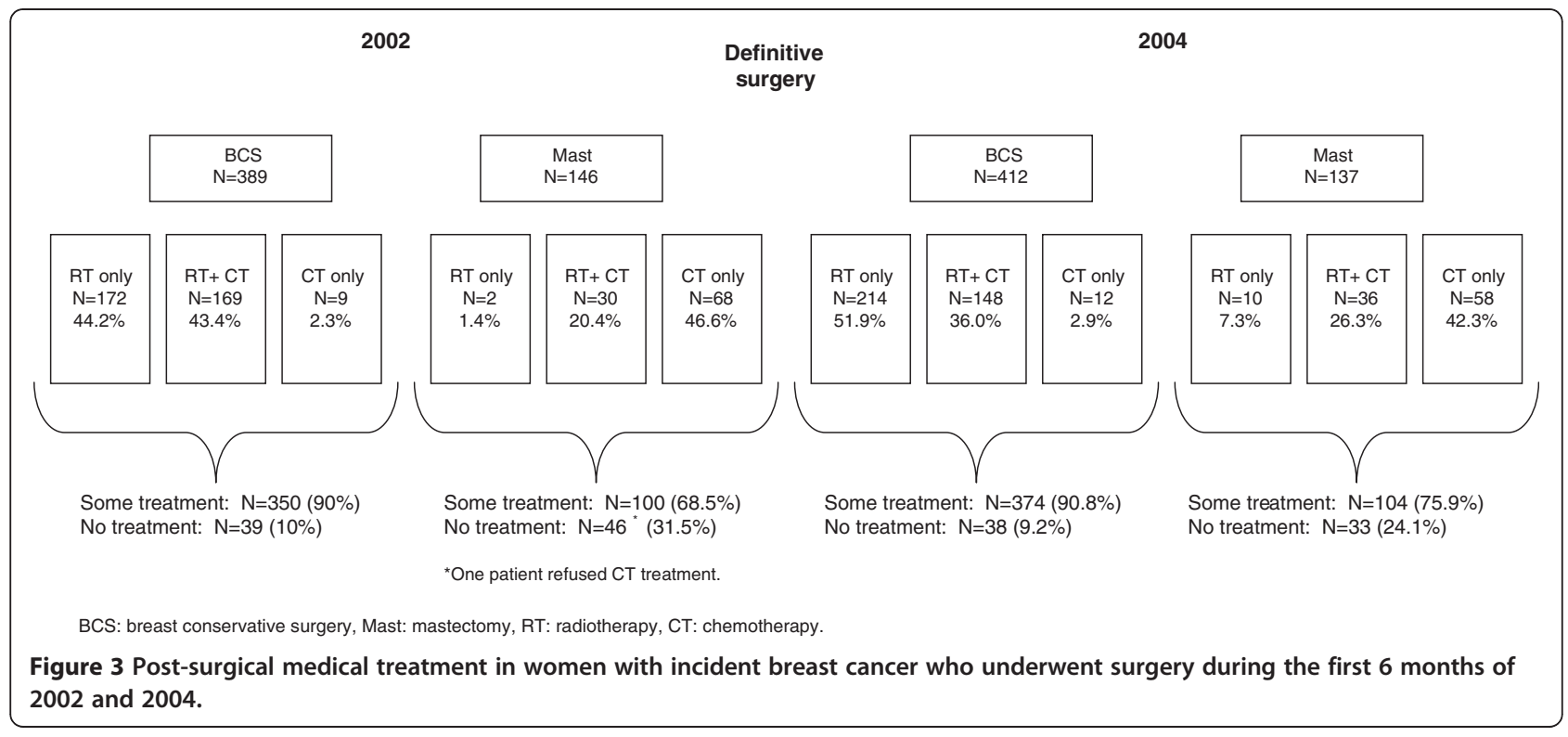

(annual case load $<50=55 \%$ increase, $50-149=40 \%$ increase, $\geq 150=41 \%$ increase) (Table 6).

\section{Survival analyses}

Between 2002 and 2010, a total of 101 deaths were identified: 52 women in the pre-PGL and 49 in the post-PGL group (90.4\% and $90.1 \%$ crude 5-year survival, respectively).

We did not find any change in the risk of mortality in the post-PGL versus the pre-PGL group (HR 0.94, 95\%CI $0.56-1.56$ adjusted for age, clinical stage and surgical unit annual case load).

\section{Discussion}

This study evaluated compliance with PGL for breast cancer by comparing cases treated before and after their introduction. Patient and tumor characteristics were comparable between pre-PGL and post-PGL patients and the same methods of ascertainment and data collection were used.

Table 3 Post-surgical chemotherapy by risk of distant metastasis according to ER and lymph node status, 2002 and 2004, Piedmont Region, Italy

\begin{tabular}{cccc}
\hline & $\begin{array}{c}\text { Low Risk* } \\
\text { N and \% }\end{array}$ & $\begin{array}{c}\text { Medium-to-high risk§ } \\
\text { N and \% }\end{array}$ & $\begin{array}{c}\mathbf{p} \\
\text { value }\end{array}$ \\
\hline $\mathbf{2 0 0 2}$ & $6 / 42$ & $268 / 409$ & 0.04 \\
& $(14.3 \%)$ & $(65.5 \%)$ & \\
$\mathbf{2 0 0 4}$ & $1 / 71$ & $252 / 396$ & \\
& $(1.4 \%)$ & $(63.6 \%)$ &
\end{tabular}

ER: estrogen receptor.

* ER-positive and size $\leq 2 \mathrm{~cm}$ and grading=1 with negative lymph node status. $\S$ ALL patients with positive lymph node status or estrogen receptor-negative or size $>2 \mathrm{~cm}$ or grading $\geq 2$ regardless of lymph node status.
In a previous report we investigated the distribution, implementation and evaluation of PGL among clinicians who treat breast cancer in the Piedmont Region. We found that approximately $90 \%$ of surgeons, gynecologists, oncologists and radiologists working in the field (70.2\% of those who responded to the questionnaire), were aware of PGL within 1 year of their release, and generally had a positive attitude to change their practice accordingly [3].

In this population-based study we examined clinical practice patterns before and after the introduction of PGL. We and observed good compliance with PGL before their release, and a weak increase in the number of medical decisions that complied with them after their release.

The literature contains several examples of situations in which clinical practice guidelines on breast cancer treatment contributed to an improvement in quality of care $[6,7,11,12]$, but very few of them included a comparison of clinical practice prior to the release of the guidelines [6], or examined this practice at a population level [7].

\begin{tabular}{|c|c|c|c|}
\hline & $\begin{array}{c}\text { Estrogen } \\
\text { receptor-positive }\end{array}$ & $\begin{array}{c}\text { Estrogen } \\
\text { receptor-negative }\end{array}$ & $\begin{array}{c}\mathrm{p} \\
\text { value }\end{array}$ \\
\hline & $\mathrm{N}$ and $\%$ & $\mathrm{~N}$ and $\%$ & \\
\hline \multirow[t]{2}{*}{2002} & $319 / 338$ & $23 / 117$ & $<0.01$ \\
\hline & (88.0\%) & (19.6\%) & \\
\hline \multirow[t]{2}{*}{2004} & $332 / 354$ & 10/92 & \\
\hline & (89.8\%) & $(10.8 \%)$ & \\
\hline
\end{tabular}


Table 5 Achievement of Piedmont Region clinical practice guidelines quality-of-care standards in 2002 and 2004, and effects of year, Piedmont Region, Italy

\begin{tabular}{|c|c|c|c|c|c|}
\hline & \multicolumn{4}{|c|}{ Results } & \multirow{3}{*}{$\begin{array}{l}\text { Effect of year (adjusted* OR and } 95 \% \mathrm{Cl} \text { ) } \\
\qquad 2002 \text { vs } 2004\end{array}$} \\
\hline & \multicolumn{2}{|c|}{2002} & \multicolumn{2}{|c|}{2004} & \\
\hline & $\mathrm{N}$ and $\%$ & Missing & $\mathrm{N}$ and $\%$ & Missing & \\
\hline \multirow{2}{*}{$\begin{array}{l}\% \text { of malignant lesions with cytological or } \\
\text { histological pre-operative diagnosis }\end{array}$} & $297 / 513$ & 48 & $329 / 495$ & 65 & $0.64(0.49-0.85)$ \\
\hline & $(57.9 \%)$ & $(8.6 \%)$ & $(66.5 \%)$ & (11.6\%) & \\
\hline \multirow[t]{2}{*}{$\%$ BCS in pT1, unifocal } & $231 / 268$ & 0 & $253 / 272$ & 0 & $0.41(0.22-0.75)$ \\
\hline & $(86.2 \%)$ & & $(93.0 \%)$ & & \\
\hline \multirow[t]{2}{*}{$\%$ BCS with free margins (> $1 \mathrm{~mm}$ ) } & $312 / 368$ & 33 & $348 / 387$ & 33 & $0.65(0.41-1.01)$ \\
\hline & $(84.8 \%)$ & $(8.2 \%)$ & $(89.9 \%)$ & $(7.9 \%)$ & \\
\hline \multirow[t]{2}{*}{$\%$ single surgery after diagnosis } & $332 / 369$ & 4 & $342 / 376$ & 8 & $1.02(0.24-5.91)$ \\
\hline & $(90.0 \%)$ & $(1.1 \%)$ & (91.0\%) & $(2.1 \%)$ & \\
\hline \multirow[t]{2}{*}{$\%$ frozen section in lesions $\leq 10 \mathrm{~mm}$} & $55 / 97$ & 1 & $85 / 113$ & 0 & $0.32(0.16-0.65)$ \\
\hline & $(56.7 \%)$ & $(1.0 \%)$ & $(75.2 \%)$ & & \\
\hline \multirow{2}{*}{$\begin{array}{l}\% \text { of patients with invasive lesion treated } \\
\text { with axillary clearance or SLN technique }\end{array}$} & $433 / 468$ & 0 & $460 / 478$ & 1 & $0.28(0.10-0.85)$ \\
\hline & $(92.5 \%)$ & & $(96.2 \%)$ & $(0.2 \%)$ & \\
\hline \multirow{2}{*}{$\begin{array}{l}\% \text { of patients treated with axillary } \\
\text { clearance with }>9 \text { lymph nodes }\end{array}$} & $317 / 336$ & 4 & $245 / 268$ & 4 & $1.71(0.89-3.31)$ \\
\hline & $(94.3 \%)$ & $(1.2 \%)$ & $(91.4 \%)$ & $(1.5 \%)$ & \\
\hline \multirow[t]{2}{*}{$\%$ with NO dissection among CIS patients** } & $115 / 123$ & 0 & $98 / 104$ & 0 & $0.60(0.14-2.50)$ \\
\hline & $(93.5)$ & & $(94.2 \%)$ & & \\
\hline \multirow[t]{2}{*}{ \% correct identification of SLN } & $118 / 126$ & 30 & $215 / 219$ & 54 & $0.46(0.18-1.05)$ \\
\hline & $(93.6 \%)$ & $(19.2 \%)$ & (98.2\%) & $(19.8 \%)$ & \\
\hline \multirow[t]{2}{*}{$\%$ histopathological grading available } & $455 / 462$ & 18 & $466 / 469$ & 17 & $0.34(0.08-1.42)$ \\
\hline & $(98.5 \%)$ & $(3.7 \%)$ & (99.4\%) & $(3.5 \%)$ & \\
\hline \multirow[t]{2}{*}{$\%$ hormonal receptor availability } & $455 / 471$ & 9 & $446 / 473$ & 13 & $1.80(0.94-3.48)$ \\
\hline & $(96.6 \%)$ & $(1.8 \%)$ & $(94.3 \%)$ & $(2.7 \%)$ & \\
\hline \multirow[t]{2}{*}{$\%$ immediate reconstruction after mastectomy } & $41 / 154$ & 4 & $30 / 137$ & 2 & $1.16(0.63-2.16)$ \\
\hline & $(26.6 \%)$ & $(2.5 \%)$ & $(21.9 \%)$ & $(1.4 \%)$ & \\
\hline \multirow[t]{2}{*}{$\%$ radiotherapy in patients treated with $\mathrm{BCS}$} & $341 / 389$ & 0 & $362 / 412$ & 0 & $1.01(0.60-1.5)$ \\
\hline & $(87.7 \%)$ & & $(87.9 \%)$ & & \\
\hline \multirow[t]{2}{*}{$\%$ of eligible patients that receive hormonal therapy } & $319 / 338$ & 0 & $332 / 354$ & 0 & $1.01(0.69-1.42)$ \\
\hline & $(94.3 \%)$ & & $(93.8 \%)$ & & \\
\hline
\end{tabular}

The 2004 period is the reference category for the estimation of ORs.

*Adjusted for age, educational level, clinical stage, screening provenience and surgical unit annual case load.

**Calculated on CIS lesions over-sampled group.

OR: odds ratio, BCS: breast conservative surgery, pT1: small primary tumor, CIS: carcinoma in situ, SLN: sentinel lymph node.

In 1997, Ray-Coquard and collaborators [6] conducted a study in France on 200 patients, with a before/after design, using information from medical records, and suggested significant changes in the quality of care. These changes were probably due to the introduction of clinical practice guidelines, but their results needed confirmation in a larger sample of cases.

White and collaborators [7] performed a study in Victoria, Australia in 2004 with a similar design using mailed questionnaires. All cases of early breast cancer registered in the Victorian Cancer Registry during two 6-month periods were selected and a questionnaire was sent to the relevant surgeon about patient characteristics and primary treatments. The study showed an improvement in quality of care after the introduction of clinical practice guidelines. However the study included data provided directly by surgeons from two different surveys. Significantly more surgeons completed the questionnaire in the first survey (73\%) than in the second one (52\%). The difference in the response rate between the two surveys could have introduced bias, causing a selection of those most interested in the topic, i.e., the surgeons with 


\begin{tabular}{|c|c|c|c|c|}
\hline \multirow{3}{*}{$\begin{array}{l}\text { Surgical unit } \\
\text { annual case load }\end{array}$} & \multicolumn{4}{|c|}{ Sentinel lymph node technique } \\
\hline & \multicolumn{2}{|c|}{2002} & \multicolumn{2}{|c|}{2004} \\
\hline & $\mathrm{N}$ and $\%$ & Missing & $\mathrm{N}$ and $\%$ & Missing \\
\hline \multirow[t]{2}{*}{$<50$} & $25 / 131$ & 3 & $55 / 130$ & 3 \\
\hline & (19.1\%) & $(2.2 \%)$ & $(42.3 \%)$ & $(2.2 \%)$ \\
\hline \multirow[t]{2}{*}{$50-149$} & $67 / 244$ & 7 & $113 / 250$ & 1 \\
\hline & $(27.4 \%)$ & $(2.8 \%)$ & $(45.2 \%)$ & $(0.4 \%)$ \\
\hline \multirow[t]{2}{*}{$\geq 150$} & $55 / 146$ & 8 & $95 / 149$ & 0 \\
\hline & $(37.7 \%)$ & $(5.2 \%)$ & $(63.7 \%)$ & \\
\hline \multirow[t]{2}{*}{ Overall } & $147 / 521$ & 21 & $263 / 529$ & 21 \\
\hline & $(28.2 \%)$ & $(3.9 \%)$ & $(49.7 \%)$ & $(3.8 \%)$ \\
\hline
\end{tabular}

the highest case load [13]. Furthermore, answers furnished by the physicians could have reflected not necessarily what they did in their clinical practice, but what they knew they should have done to comply with the guidelines.

In our study we collected data directly from clinical records. We found a statistically significant positive trend in two of four quality-of-care indicators concerning diagnosis. In particular we found an improvement in the two indicators that were farthest from the standard in the pre-PGL group (\% lesions with cytological/histological diagnosis before surgery and\% frozen sections in $\leq 10 \mathrm{~mm}$ lesions), but not in the two indicators that already have a good compliance with PGL (percent of histopathological grading available and percent of hormone receptor available).

We measured two important quality-of-care indicators concerning the surgical treatment of breast cancer: percent of BCS in pT1 lesions and percent of BCS performed with free margins, and the results were positive. We noticed a trend of improvement in the post-PGL group: 93\% of patients with pT1 were treated surgically with BCS in the present study. We found a similar positive trend concerning the practice of BCS over a 5-year period (2000-2004) in a previous population-based study on women with breast cancer (all ages) carried out in the Piedmont Region using administrative data [14]. Nevertheless, the percentage of single surgery after diagnosis, which was already good before PGL were released, and the percent of reconstruction after mastectomy, which was extremely low, did not show a positive trend over time.

The indicators regarding axillary surgery showed an increased proportion of patients that were treated with axillary clearance with a correct indication. The percent of patients with a clearance of $>9$ lymph nodes and the percent of dissections not performed among CIS patients did not reach the standard and did not improve after the release of PGL. Conversely, a higher number of centers performed the SLN technique, with an identification rate that reached the standard.

Finally, looking at the proportion of women who underwent SLN technique by the annual case load of the surgical unit, we found that the use of this technique increased by almost 45\% from 2002 to 2004, across all strata of surgical unit annual caseload but in particular in centers treating less than 50 breast cancer a year. This finding is clearly in contrast with the PGL recommendations that suggest the use of SLN technique only in specialized centers (surgical unit annual case load $>50$ ). The increase of use of SLN technique in low caseload centers need to be discouraged.

Between 2002 and 2004 the proportion of women who received radiotherapy after breast cancer surgery $(87.7 \%$ in 2002 and $87.9 \%$ in 2004) was stable, though still far from the standard of $95 \%$. In a previous populationbased study in Piedmont, the presence of a radiotherapy unit within the same hospital where the surgical procedure was performed was associated with a higher probability of receiving radiotherapy after discharge. The presence of a radiotherapy unit in the hospital also correlated with the case load and specialization of the surgical unit [14].

In the analyses of post-surgical medical treatment we found a decrease in the percent of patients with invasive lesions at medium-to-high risk of distant metastasis who received chemotherapy after the introduction of PGL. Furthermore, we observed a decrease in the percent of patients with invasive lesions and low risk of distant metastasis who received inappropriate chemotherapy. The percent of patients who received hormonal therapy was stable in the group with positive estrogen receptor status, and the incorrect prescription of hormonal treatment in estrogen receptor-negative women dramatically decreased in the post-PGL group.

Apart from the introduction of PGL, the positive trend in some of quality-of-care indicators can be partly attributed to the increased proportion of breast cancer cases diagnosed through the regional screening program. In fact, the patients who were diagnosed in the context of the screening program were usually referred to a surgical unit with a high annual case load.

Underestimation of chemotherapy, radiotherapy and/or hormonal therapy was possible given that these treatments are administrated at a different hospital admission than that for the surgical treatment, or even on an ambulatory basis. The information we collected about post-surgical medical treatment was the result of record-linkages between breast cancer patients and the HDR database, radiotherapy outpatient record database and pharmaceutical prescription record database. Such linkages can generate omissions that are likely to be random, so the resulting bias would be conservative. 
Finally, we did not see changes in the survival rate between pre-PGL and post-PGL groups. In fact, the majority of PGL recommendations were oriented to avoid invasive surgery, over-treatment, recurrence, or patient anxiety (i.e., avoid mastectomy in pT1 unifocal, avoid more than one surgery after diagnosis, avoid dissection in CIS patients). Very few recommendations were formulated to improve survival (i.e., to measure hormonal receptor availability, to perform radiotherapy in patients treated with BCS, to perform axillary clearance or SLN technique in patients with invasive lesions). Moreover the indicators more related to survival, with the exception of axillary clearance, showed only a negligible improvement after the introduction of PGL. Finally it is possible that, since breast cancer is generally characterized by long survival, a small improvement in survival will manifest itself only with longer follow-up.

The population-based approach of the study ensures that selection bias was minimal and that the results can be considered representative of the entire Piedmont Region.

\section{Conclusions}

The results suggest that the majority of quality-of-care indicators changed in the expected direction after the introduction of PGL, even if only 4 out of 14 reached statistical significance. Statistical significance was registered in particular in the indicators that were far from achieving the standard. Our results highlight the need to continue to improve breast cancer care and to measure the correct adherence to PGL.

In a previous study we evaluated the distribution, implementation and acceptance of PGL among medical doctors, and in the present paper we present the results based on quality-of-care indicators before and after the introduction of PGL. The ultimate evaluation must involve long-term outcome studies on recurrence and mortality to assess the real impact of PGL on patient care, and economic evaluations to assess treatment choices.

\section{Abbreviations \\ PGL: Piedmont guidelines; HDR: Hospital discharge records; N: Number; BCS: Breast conservative surgery; CIS: Carcinoma in situ; ORadj: Adjusted OR; Cl: Confidence interval; SLN: Sentinel lymph node.}

\section{Competing interests}

The authors declare that they have no competing interests in connection with this paper.

\section{Authors' contribution}

CS and AP conceived of the study and co-wrote the manuscript with contributions from all other authors; RB and MPM contributed to study design and coordination of the study; SP, DC, RR, ST, AF and LM contributed to acquisition of the data and their interpretation; IB, EP, RR, DDC, MT and FR performed the statistical analysis and contributed to data interpretation; AP,
GC, NS, FM, and PV conceived of the study, contributed to data interpretation, obtaining grant funding and financial support. All authors have given final approval of the version to be published.

\section{Acknowledgements}

This study was supported by the Compagnia di San Paolo, the M.I.U.R./PRIN grant 2005068001 and the Eunice project of the European Commission. The regional program of guideline development and quality assessment has been also supported by: Piedmont Region-Regional Health Authority; Fondazione CRT-DI7 Project; Ministry of Health-Research Program ex art 12bis DL 229/99, 2006. We are grateful to Ms Aurora Di Leo, Dr Ornella Stratta, Dr Anna VignaSuria, Ms Gabriella DelMastro and Ms Elena Mura for their assistance in collecting data. Finally we thank you all the Coordinators of the Piedmont breast cancer screening program.

\section{Author details}

${ }^{1}$ Cancer Epidemiology Unit, San Giovanni Battista Hospital, CPO Piemonte and University of Turin, Turin, Italy. ${ }^{2}$ Cancer Epidemiology Unit, San Giovann Battista Hospital, CPO Piemonte, Turin, Italy. ${ }^{3}$ Screening Centre, Department of Cancer Prevention, San Giovanni Battista Hospital, CPO Piemonte, Turin, Italy. ${ }^{4} \mathrm{HuGeF}$ Foundation, Via Nizza 52, 10126, Torino, Italy. ${ }^{5}$ Department of Epidemiology and Biostatistics, School of Public Health, Imperial College, London, UK. ${ }^{6}$ Cancer Epidemiology Unit, San Giovanni Battista Hospital, Via Santena 7, 10129, Torino, Italy.

Received: 13 December 2011 Accepted: 15 January 2013

Published: 25 January 2013

\section{References}

1. Sainsbury R, Haward B, Rider L, Johnston C, Round C: Influence of clinician workload and patterns of treatment on survival from breast cancer. Lancet 1995, 345(8960):1265-1270.

2. Regione Piemonte Assessorato Sanità, Commissione Oncologica Regionale, Centro di Riferimento per l'Epidemiologia e la Prevenzione Oncologica in Piemonte: Tumore della mammella - linee guida clinico organizzative per la Regione Piemonte. 2002.

3. Sacerdote C, Pagano E, Ponti A, Rosato R, Senore C, Becattini M, Tomatis M, Piera MM, Vineis $P$, Ciccone G: The physicians' view on the utility and impact of oncological guidelines in clinical practice. A survey of Piedmont physicians. Epidemiol Prev 2007, 31(1):34-38. Italian.

4. Malin JL, Schuster MA, Kahn KA, Brook RH: Quality of breast cancer care: what do we know? J Clin Oncol 2002, 20(21):4381-4393.

5. Hillner BE, Smith TJ, Desch CE: Hospital and physician volume or specialization and outcomes in cancer treatment: importance in quality of cancer care. J Clin Oncol 2000, 18(11):2327-2340.

6. Ray-Coquard I, Philip T, Lehmann M, Fervers B, Farsi F, Chauvin F: Impact of a clinical guidelines program for breast and colon cancer in a French cancer center. JAMA 1997, 278(19):1591-1595.

7. White V, Pruden M, Giles G, Collins J, Jamrozik K, Inglis G, Boyages J, Hill D: The management of early breast carcinoma before and after the introduction of clinical practice guidelines. Cancer 2004, 101(3):476-485.

8. Baldi I, Vicari P, Di Cuonzo D, Zanetti R, Pagano E, Rosato R, Sacerdote C, Segnan N, Merletti F, Ciccone G: A high positive predictive value algorithm using hospital administrative data identified incident cancer cases. J Clin Epidemiol 2008, 61(4):373-379.

9. Ponti A, Mano MP, Distante V, Taffurelli M, Naldoni C, Zangirolami F, Vettorazzi M, Zorzi M, Pagano G, Federici A, Baiocchi D, Bordon R, Tomatis M, Mantellini P, Cataliotti L, Rosselli del Turco M, Segnan N: Audit system on Quality of breast cancer diagnosis and Treatment (QT): results from the survey on screen-detected lesions in Italy, 2003-2004. Epidemiol Prev 2006, 30(Suppl 3):59-63.

10. Goldhirsch A, Colleoni M, Gelber RD: Endocrine therapy of breast cancer. Ann Oncol 2002, 13(Suppl 4):61-68.

11. Hebert-Croteau N, Brisson J, Latreille J, Blanchette C, Deschenes L: Compliance with consensus recommendations for the treatment of early stage breast carcinoma in elderly women. Cancer 1999, 85(05):1 104-1113.

12. Olivotto A, Coldman AJ, Hislop TG, et al: Compliance with pratical guidelines for node-negative breast cancer. J Clin Oncol 1997, 15(1):216-222. 
13. Bickell NA, LePar F, Wang JJ, Leventhal H: Lost opportunities: physicians' reasons and disparities in breast cancer treatment. J Clin Oncol 2007, 25(18):2516-2521.

14. Rosato R, Sacerdote C, Pagano E, Di Cuonzo D, Baldi I, Bordon R, Ponti A, Bertetto O, Segnan N, Merletti F, Vineis P, Ciccone G: Appropriateness of early breast cancer management in relation to patient and hospital characteristics: a population based study in Northern Italy. Breast Canc Res Treat 2009, 117(2):349-356.

doi:10.1186/1472-6963-13-28

Cite this article as: Sacerdote et al:: Compliance with clinical practice guidelines for breast cancer treatment: a population-based study of quality-of-care indicators in Italy. BMC Health Services Research 2013 13:28.

\section{Submit your next manuscript to BioMed Central and take full advantage of:}

- Convenient online submission

- Thorough peer review

- No space constraints or color figure charges

- Immediate publication on acceptance

- Inclusion in PubMed, CAS, Scopus and Google Scholar

- Research which is freely available for redistribution 